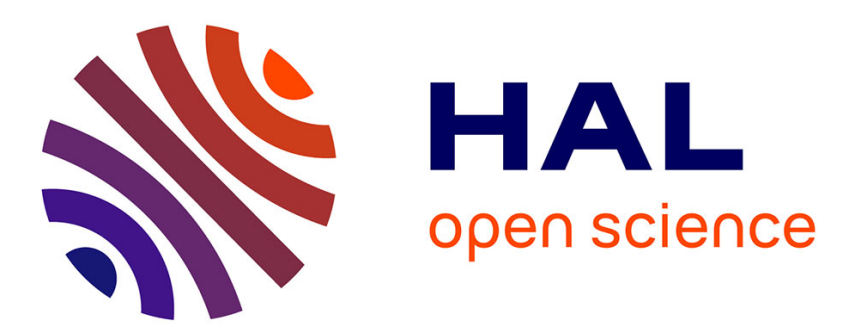

\title{
La communication environnementale légitimée pour éduquer au développement durable
}

\author{
Aurélie Zwang
}

\section{To cite this version:}

Aurélie Zwang. La communication environnementale légitimée pour éduquer au développement durable. Questions de communication, 2017, 32, pp.105-124. 10.4000/questionsdecommunication.11448. hal-03130773

\section{HAL Id: hal-03130773 \\ https://hal.science/hal-03130773}

Submitted on 3 Feb 2021

HAL is a multi-disciplinary open access archive for the deposit and dissemination of scientific research documents, whether they are published or not. The documents may come from teaching and research institutions in France or abroad, or from public or private research centers.
L'archive ouverte pluridisciplinaire HAL, est destinée au dépôt et à la diffusion de documents scientifiques de niveau recherche, publiés ou non, émanant des établissements d'enseignement et de recherche français ou étrangers, des laboratoires publics ou privés. 


\section{Questions de communication}

32 | 2017

Environnement, savoirs, société

\section{La communication environnementale légitimée pour éduquer au développement durable}

De l'attente envers l'autorité éditoriale comme rapport de l'École à la trivialité

Legitimating Environmental Communication to Educate for Sustainable

Development: Expectations of Editorial Authority as the School's Relationship to

Triviality

\section{Aurélie Zwang}

\section{(2) OpenEdition}

\section{Journals}

Édition électronique

URL : http://journals.openedition.org/questionsdecommunication/11448

DOI : 10.4000/questionsdecommunication. 11448

ISSN : 2259-8901

Éditeur

Presses universitaires de Lorraine

\section{Édition imprimée}

Date de publication : 31 décembre 2017

Pagination : 105-124

ISBN : 9782814305076

ISSN : 1633-5961

Référence électronique

Aurélie Zwang, « La communication environnementale légitimée pour éduquer au développement durable», Questions de communication [En ligne], 32 | 2017, mis en ligne le 31 décembre 2019, consulté le 04 janvier 2021. URL : http://journals.openedition.org/questionsdecommunication/11448; DOI : https://doi.org/10.4000/questionsdecommunication.11448 


\title{
LA COMMUNICATION ENVIRONNEMENTALE LÉGITIMÉE POUR ÉDUQUER AU DÉVELOPPEMENT DURABLE DE L'ATTENTE ENVERS L'AUTORITÉ ÉDITORIALE COMME RAPPORT DE L'ÉCOLE À LA TRIVIALITÉ
}

\begin{abstract}
Résumé. - L'article s'appuie sur les résultats d'une recherche interdisciplinaire axée sur la légitimation d'expositions itinérantes comme médias pour éduquer au développement durable. Leur mise en circulation entre l'extérieur et l'intérieur de l'École dépend peu de critères pédagogiques et donne aux élèves accès à des productions peu discriminées pour l'usage scolaire. Réduites à des panneaux, leur analyse, fondée sur l'iconicité du texte et sur les propriétés énonciatrices des plages visuelles, couplée à celle des logiques de légitimation éducative, met en évidence que les attentes des cadres de l'Éducation nationale à propos des institutions productrices, soit des autorités éditoriales, sont à rapprocher de leurs énonciations pour comprendre quels savoirs, valeurs et représentations socioenvironnementaux sont légitimés, dans un rapport de l'École à la trivialité qu'il serait pertinent de considérer en sciences de l'éducation.
\end{abstract}

Mots clés. - expositions itinérantes, analyse sémiotique, autorité éditoriale, trivialité, communication de l'environnement, éducation au développement durable 
$\grave{A}$

l'instar d'autres sphères sociales et selon le même schème descendant, l'École a intégré le développement durable comme mode de pensée des problématiques écologiques contemporaines par l'introduction, dès 2004, d'une éducation au développement durable (EDD). Ce champ éducatif est bâti sur un objet théorique protéiforme, fusionnant les enjeux environnementaux, sociaux et économiques dans un « référent social [...] signifi[ant] quelque chose pour tous » (Krieg-Planque, $2010: 6$ ) et à propos duquel l'essor des productions médiatiques et culturelles, depuis les années 2000 (Libaert, 20 I 6), met l'École face à un champ polyphonique et hétérogène de médias éducatifs potentiels (D'Almeida, Jalenques-Vigouroux, 2007). Parmi eux, les expositions itinérantes ont été promues par le ministère de l'Éducation nationale (EN) comme support favorisant la transdisciplinarité, avec force déclarations sur la nécessité d'une correspondance de contenu entre ces médias et les objectifs éducatifs de l'institution.

L'autorisation symbolique de leur introduction dans l'espace scolaire est ici examinée dans le cadre des rapports de l'École à la trivialité, concept forgé parYves Jeanneret (2008, 2014) qui permet de penser le partage et la circulation des idées, via les supports matériels de communication, en interrogeant la perpétuelle transformation des « êtres culturels », complexes liant objets, textes et représentations, qui se chargent de valeurs au cours de leurs parcours communicationnels. L'attribution d'une valeur éducative à ces médias, une fois choisis par des cadres de l'EN, sans être une obligation pour permettre leur utilisation compte tenu de la liberté pédagogique laissée aux enseignants, est suffisamment significative pour qu'elle soit chargée d'un sens prescriptif. Or, en légitimant ces médias, quels savoirs, valeurs et représentations relatifs aux problématiques socio-environnementales l'institution scolaire laisse-t-elle pénétrer dans ses murs?

Par une approche orientée vers les contenus, j'ai déjà montré que, loin d'être circonscrits par la discrimination pédagogique annoncée, les objectifs éducatifs des expositions itinérantes ne correspondent pas à ceux des textes officiels de l'EDD (Zwang, 2016). Aussi, cette dissonance établie, en quoi une analyse communicationnelle des logiques de légitimation éducative à l'œuvre permet-elle de l'expliciter? Après avoir présenté le rôle, les voies et les logiques de légitimation éducative dont le dénominateur commun est l'identité des producteurs d'expositions, j'analyserai ces dernières à l'aune du contrat de communication qui lie les attentes des cadres de l'EN aux intentions communicationnelles des producteurs retenus à travers plusieurs exemples (Le Marec, 2007). In fine, je montrerai que considérer la communication sur l'environnement dans ce contexte permet de comprendre pourquoi les expositions retenues, au-delà de la limite inhérente à leur format de documents, ne donnent à voir aux élèves que des rapports dépolitisés à l'environnement où injonctions et bonnes intentions prévalent, sans place pour une mise en débat des responsabilités partagées. 


\section{Des expositions itinérantes légitimées pour éduquer au développement durable}

En milieu scolaire, des médias sont convoqués au quotidien pour étayer la relation pédagogique entre enseignants et élèves. Si certains ont été élaborés avec une finalité didactique - à l'instar des manuels scolaires -, la plupart d'entre eux ne deviennent « éducatifs » qu'à la suite de leur incorporation dans le système de formation (Mœglin, 2004). Lorsque les cadres de l'EN déploient des logiques électives pour les usages de l'ensemble des communautés scolaires, la reconnaissance pédagogique attribuée au média est une autorisation symbolique de circulation matérielle dans l'enceinte scolaire. Et, même si le corps enseignant a toute liberté de s'en affranchir, cette reconnaissance institutionnelle est recherchée par les éditeurs car elle leur garantit une diffusion en milieu formel. C'est pourquoi, au XIX siècle, la maison d'édition Deyrolle, alors grand fournisseur de matériel scolaire, utilisait déjà comme argument de vente de son musée scolaire constitué de planches murales le fait qu'il avait été « adopté par le Ministère de l'instruction publique » (Deyrolle, I 898 : 27).

En 2006, le tirage à plus de 50000 exemplaires d'un kit pédagogique fondé sur les photographies de Yann Arthus-Bertrand' est l'exemple type du rôle d'opérateur symbolique joué par la légitimation éducative : de clichés esthétiques sur les grilles du Luxembourg, ces instantanés aériens sont devenus des imageries éducatives une fois estampillés par l'institution scolaire. De plus, cette vaste opération - intitulée Le Développement durable, pourquoi ? Un exposition pédagogique pour chaque école de France $^{2}$ - a consacré l'affichage mural de documents traitant de sujets socio-environnementaux comme levier pour généraliser l'EDD en milieu formel. Dès lors, nombre d'autres productions industrielles nommées « expositions » par leurs concepteurs ont acquis une valeur pour l'EDD, le plus souvent après leur parution.

La légitimité se concrétise soit par une coproduction avec l'EN, soit par une sélection via les établissements du réseau de documentation pédagogique, ou encore par une publicisation sur des sites officiels, le plus souvent au sein d'une rubrique de « ressources pédagogiques », en faisant également des liens vers des sites de producteurs. 196 expositions éditées entre 1990 et 201 I ont été identifiées comme médias éducatifs reconnus pour l'EDD. Elles ont été dénombrées à partir des bases de données des 3 I centres régionaux de documentations pédagogiques, des rubriques de ressources pédagogiques du pôle de compétence national en $\mathrm{EDD}^{3}$ et des 21 sites internet académiques spécialisées en EDD ainsi que sur les sites des producteurs signalés comme pourvoyeurs d'expositions pour cette

\footnotetext{
I Accès : http://www.ledeveloppementdurable.fr/developpementdurable/. Consulté le 24/I I/20 I7.

2 Accès : http://wnw.ledeveloppementdurable.fr/developpementdurable/page/operation.html. Consulté le 24/ I I/2017.

3 Accès : https://crdp.ac-amiens.fr/edd/. Consulté le 29/07/2017.
} 
éducation. II ne s'agit donc en aucun cas d'un recensement des expositions circulant dans les établissements scolaires, mais d'un panorama des possibles, fondé sur des recommandations au sein d'un milieu professionnel.

D'expositions, elles ont davantage le nom pour leur prétention de monstration que pour leurs attributs muséologiques. Leur « intentionnalité constitutive » (Davallon, I999: I I) est floue ; ce que montre la plasticité du vocable employé : les différentes éditions du Développement durable, pourquoi ?4 sont qualifiées tout à la fois « d'expositions pédagogiques $»^{5}$ qui contiennent des « affiches » ou « d'opération "Posters école" " ${ }^{6}$ quand la maison d'édition Deyrolle qualifie les

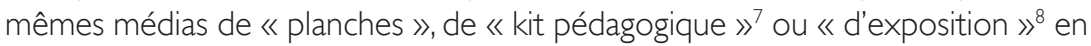
fonction du lieu de présentation. Leur caractère itinérant a pour conséquence une homogénéisation des supports matériels exposés, soit des panneaux à $92 \%$, rarement reliés par une trame narrative (66\% n'en possèdent pas, soit I I 5 sur 174 expositions lisibles). Ces médias sont donc essentiellement composés d'affiches dissociables les unes des autres. Inclassables dans la typologie établie par Jean Davallon (1999), c'est-à-dire qu'elles ne relèvent ni de la muséologie d'objets, ni tout à fait de la muséologie de savoirs ou de celle de points de vue, ces expositions environnementales ont comme principal point commun d'être le fruit d'une muséologie à approche thématique (Le Marec, 20l I).

Parmi ces thématiques, les plus fréquentes sont le développement durable, l'eau, les déchets, la biodiversité, l'énergie, le changement climatique et les milieux qui représentent à elles seules $73 \%$ du corpus, soit 110 expositions. Les productions majoritairement retenues sont issues d'organisations publiques (à hauteur de $45 \%$ telles l'Agence de l'environnement et de la maitrise de l'énergie (Ademe) (22/I I 0 expositions), I'agence de l'eau Adour Garonne (7), I'Institut de recherche pour le développement (IRD - 6), l'Institut national de recherche agronomique (Inra - 5) ou encore le ministère de l'Environnement et du Développement durable (MEDD 4), à côté desquelles le secteur privé est essentiellement représenté par deux agences d'expositions (C'est comme vous voulez,7 ; et Double Hélice, 5), la fondation GoodPlanet (5) et Deyrolle. Le monde associatif, quant à lui, est très hétérogène et clairsemé avec presque autant d'expositions différentes que d'associations ( 13 pour 12 associations) à l'exception de celles de l'Agence régionale de l'environnement de Haute-Normandie ( I I) et de la Maison des sciences et du développement durable (4). La plupart des 40 organisations ayant conçu les expositions légitimées

\footnotetext{
${ }^{4}$ Entre 2006 et 201 I, la fondation GoodPlanet a édité cinq kits d'une vingtaine d'affiches sur les thèmes du développement durable (2006), de la biodiversité (2007), de l'énergie (2009), de l'eau (20l0) et de la forêt (20I I), toutes pour le public scolaire.

${ }^{5}$ Accès : http://www.ledeveloppementdurable.fr/developpementdurable/. Consulté le 03/08/20 I7.

${ }^{6}$ Accès : http://eduscol.education.fr/cid50760/l-eau-une-ressource-vitale.html. Consulté le 03/08/20 I7.

7 Accès : https://www.deyrolle.com/la-vocation-pedagogique/de-deyrolle-a-dpa/les-planches/les-kitspedagogiques. Consulté le 03/08/2017.

${ }^{8}$ Accès : https://www.deyrolle.com/ ?option =com_content\&view =article\&id $=191$ :les-expositionsen-france\&catid = 46 : deyrolle-pour-l-avenir. Consulté le 03/08/2017.
} 
du corpus a des missions de diffusion, d'information ou de sensibilisation vers le grand public. La prédominance de l'Ademe, et du MEDD, explique qu'environ $20 \%$ d'entre elles relève de campagnes publiques de communication, mises à disposition gratuitement, orientées soit vers la réduction des déchets, soit vers l'adoption de pratiques vertueuses pour les semaines du développement durable de 2008, 2009 et 20 I0. En définitive, seules $26 \%$ des productions du corpus ont été pensées pour un usage scolaire. Cela revient à dire que, pour la grande majorité d'entre elles, les intentions communicationnelles des producteurs ne sont pas orientées directement vers le monde scolaire avec les enseignants et leurs élèves comme public cible.

\section{Une légitimation éducative comme intermédiaire de la rencontre entre média et public scolaire}

Cette configuration est différente de celle existant, par exemple, entre des éditeurs scolaires et les enseignants susceptibles de retenir leurs livres puisque la relation entre le média, l'enseignant et ses élèves est ici une résultante de la légitimation éducative des cadres de l'EN qui joue donc un rôle d'intermédiaire. Pour tenter de comprendre comment cette intermédiation s'organise, des entretiens semidirectifs ont été réalisés en 20 I I avec 17 cadres de l'EN de la Direction générale de l'enseignement scolaire et des académies d'Amiens, Créteil, Nancy-Metz, Paris, Poitiers, Rouen et Versailles, tous en charge, avec des fonctions différentes, de I'EDD : hauts fonctionnaires, inspecteurs généraux et régionaux, documentalistes et cadres dirigeants du réseau de documentation. Ces rencontres ont d'abord permis d'établir que l'organisation socio-économique des échanges entre extérieur et intérieur de l'École est en grande partie orientée par ses missions de service public, parmi lesquelles la gratuité pour l'usager est un impératif. Le modèle du club (Mœglin, 2004) régit en grande partie les transactions : un abonnement modique auprès du réseau de documentation permet potentiellement à un établissement scolaire de disposer de plusieurs expositions à l'année. Pour assurer ces prêts, le réseau choisit avec parcimonie des productions coûteuses acquises auprès d'agences d'expositions et acquiert davantage de productions à bas prix, voire gratuites, issues d'organisations souhaitant diffuser leurs productions à un très large public. Ces entretiens ont également permis de mettre en évidence trois logiques de légitimation : une légitimation scientifique fondée sur le rayonnement scientifique ou expert octroyé par l'institution au producteur ; une légitimation institutionnelle reposant sur la conformité des producteurs aux valeurs et aux finalités de l'EDD ; une légitimation politique issue d'une décision du cabinet ministériel de faire entrer dans l'espace scolaire des productions de certains éditeurs. La part relative de ces logiques dépend des fonctions - en effet, l'expression cadres de l'EN ne recouvre pas une entité homogène, même si elle est employée ici pour appuyer le point de vue institutionnel de leurs actions. Ainsi les hauts fonctionnaires se voient-ils davantage impliqués dans la légitimation politique. Cependant, ces logiques ont comme point commun de reposer sur l'identité du producteur, de sorte que 
« les contours des espaces de légitimités scolaires, dans lesquels les expositions itinérantes sont autorisées à circuler, sont dessinés par les représentations des cadres de l'EN sur leurs producteurs » (Zwang, 2016:42). L'examen pédagogique des contenus n'est donc pas prioritaire, avec pour conséquence le fait que les productions choisies correspondent peu aux savoirs et aux pratiques promus par les cadres prescriptifs de l'EDD scolaire.

D'un point de vue didactique, l'analyse pourrait s'arrêter à ce constat critique d'une inadéquation de la politique documentaire avec les objectifs éducatifs officiellement visés. Mais, dans la continuité de ces travaux, en cherchant à « concilier analyse des objets et prise en compte des représentations » (Jeanneret, Patrin-Leclere, 2004 : 136), je souhaite montrer comment une approche communicationnelle permet de mettre en rapport les présupposés sur les contenus liés à l'identité du concepteur avec les formes énonciatives de ces productions. Autrement dit, je souhaite montrer que des contrats de communication implicites déterminent en grande partie le rapport de l'École à la trivialité en tant que carrefour (trivium) de la vie sociale dont la nature et la fonction sont de sélectionner et d'organiser des savoirs (Jeanneret, 1999).

\section{Un rapport de l'École à la trivialité inscrite dans des contrats de communication entre cadres de l'EN et autorité éditoriale}

Si les producteurs cherchent à diffuser très largement leurs expositions, la rencontre directe entre le média et le public scolaire n'est pas spécifiquement anticipée, sauf pour le quart de productions du corpus ciblant les scolaires. Pour ce dernier, on peut supposer qu'il existe des « contrats de lecture » c'est-à-dire, tel que démontré par Eliseo Verón (1985), un dispositif d'énonciation matérialisant une proposition de relation entre un énonciateur et le public qu'il cible, ici le public scolaire. Mais, d'une part, la légitimation éducative est un intermédiaire et, d'autre part, certaines productions sont légitimées sans lecture du média, via des liens hypertextes sur la base unique de l'identité des producteurs. Autrement dit, à l'instar du contrat de communication institutionnel en milieu muséal (Le Marec, 2007 : 193), les rapports aux expositions légitimées se construiraient sur une «culture commune des processus de communication partagée » qui lierait de façon implicite les attentes des cadres de l'EN aux producteurs d'expositions qu'ils privilégient, c'est-à-dire sur la base de l'anticipation confiante de leurs propositions communicationnelles. Ceci implique de mettre en regard les logiques de légitimations scientifique, institutionnelle et politique avec les logiques communicationnelles à l'œuvre dans les productions légitimées pour considérer de façon opératoire les deux versants de l'intermédiation qui structure la circulation des idées, des valeurs et des représentations entre l'extérieur et l'intérieur de l'École. 
Sans revenir en détail sur les entretiens qui ont permis de déduire les logiques de légitimation (Zwang, 2016) et à propos desquelles je n'extrairai que quelques verbatim utiles à la cohérence du propos, il faut présenter les ancrages de mon analyse communicationnelle. J'ai spécifiquement considéré la « part formelle instituante » (Souchier, $2007: 31$ ) de l'écrit qui s'incarne dans l'énonciation éditoriale (Souchier, 1998) : des contributions multiples, qui semblent s'effacer derrière un auteur, sont à l'origine de l'élaboration collective d'une « image du texte », au sens matériel (mise en page, format, typographie, etc.) et au sens figuré (du point de vue de sa notoriété), si bien que, « à des degrés divers, ces traces ou "marques d'énonciation éditoriale" façonnent et constituent l'identité du texte. Elles déterminent donc les conditions de sa réception » (ibid. : 142). L'énonciation éditoriale ne saurait se limiter au texte dans le sens où la mise en forme graphique des énoncés dans l'interpénétration du visible et du lisible au sein du registre scriptovisuel (Jacobi, 2005) non seulement donne une iconicité mais contribue aussi à forger l'image de l'instance productrice et donc des rapports de légitimités. En muséologie, Joëlle Le Marec et Roland Topalian (2003 : I 5) ont considéré que si, dans une exposition, les contributeurs sont clairement identifiés - ce qui est le rôle de l'ours -, ils s'unissent néanmoins derrière « l'institution qui a le pouvoir de mettre en forme le système de communication et d'assumer l'autorité éditoriale ». Dans les médias étudiés, la responsabilité éditoriale du contenu est tangible par l'emploi de logotypes, mais l'autorité éditoriale s'exprime également par des modalités communicationnelles travaillées dans le registre scriptovisuel.

Des empreintes institutionnelle et culturelle signent visuellement des intentionnalités communicationnelles, approchées par une « sémiotique ouverte » (Boutaud, Verón, 2007). Au sein de chaque panneau, considéré en tant qu'unité de sens autonome, l'iconicité du texte donne fréquemment à voir un titre, un chapeau, des intertitres et des blocs de textes distincts pouvant présenter des saillances par la police, la casse, la graisse ou la couleur ; une rhétorique visuelle (Catellani, 2016) se manifestant également via les éléments du registre scriptovisuel employés - tableaux, graphiques, schémas, cartes, dessins, etc. - considérés pour leurs propriétés communicationnelles. La matérialité graphique de chaque document ${ }^{9}$ permet de déterminer un ou des thème(s) - ce dont il est question - et le ou les angle(s) d'approche(s) du thème, c'est à dire la ou les façon(s) dont il est cadré, en dégageant principalement une tendance. Par exemple, au sein d'une grande thématique, comme l'eau, des thèmes sont traités, tels la gestion de l'eau, qui peuvent être approchés selon différents angles : historique, scientifique, comportementaliste, technique, etc. Sans valeur absolue, ces catégories ont été établies de façon empirique à l'aune du corpus étudié et prennent sens dans une démarche comparative, fondée sur un traitement statistique des données, en particulier sur des analyses factorielles de correspondance $^{10}$ (AFC), approfondies qualitativement.

\footnotetext{
${ }_{9}$ Chaque exposition du corpus comprend en moyenne 12 panneaux.

${ }^{10}$ L'AFC est une méthode d'analyse de données qualitatives fondée sur le test du Khi². La distance entre les données observées et les données théoriques est projetée graphiquement sur des axes, appelés
} 
Ainsi les logiques de légitimation des cadres de l'EN en EDD peuvent être interrogées à l'aune de leurs rapports aux autorités éditoriales en ce que celles-ci façonnent des relations via des énonciations propres comme expressions culturelles et institutionnelles de leur rapport aux problématiques socio-environnementales.

\section{Logiques de légitimation et énonciations éditoriales: des contrats de communications en question}

Autour des autorités éditoriales, se nouent les contrats de communication qui conduisent les cadres de l'EN à scolariser certaines productions par anticipation de ce que celles-ci comporteraient comme traitement des thématiques socioenvironnementales. Mais dans cette relation, y a t-il toujours une adéquation ? A contrario, en quoi cet implicite communicationnel peut-il mettre en évidence des attentes parfois non exprimées par les cadres de l'EN? L'hétérogénéité du corpus exclut que je rende compte de tous les rapports de légitimités à l'origine du choix des 110 expositions étudiées. Aussi rapprocherai-je les logiques de légitimation avec les principales tendances déduites de l'AFC entre les types de producteurs et les angles d'approche des thèmes pour les sept thématiques les plus fréquentes (figure I) auquel j'ajouterai l'exemple de Deyrolle comme révélateur des attentes éducatives de certains hauts cadres de l'institution scolaire.

Figure I. AFC entre les types de producteurs et les angles d'approche des thèmes pour les sept thématiques étudiées $(n=110)^{11}$

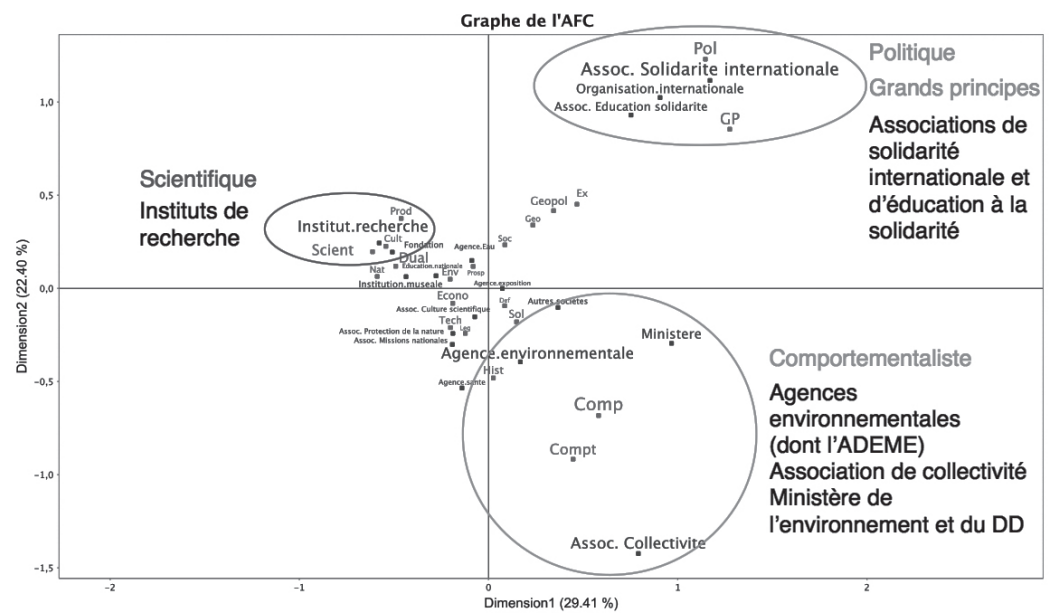

axes d'inertie. Ils permettent d'opposer les points qui ont les plus grandes distances, c'est-à-dire les plus grandes différences.

" Comp : comportementaliste ; Compt : comptable ; Hist : Historique ; Pol : politique ; GP : grands principes ; Prod : producteur ; Scient : scientifique. 


\section{L'énonciation de l'expertise ou le malentendu de la légitimation scientifique}

La légitimation scientifique est donnée par les cadres de l'EN en fonction du savoir académique que les productions retenues sont censées contenir : « On sait qu'on a la caution scientifique, donc on sait que le contenu de l'exposition sera fiable » (EN 17) ${ }^{12}$. C'est dans cette perspective que les productions des instituts de recherche sont choisies, le plus souvent de façon indirecte via des liens vers les sites des instituts de recherche scientifique avec comme recommandation de pouvoir s'appuyer sur leurs expositions pour l'EDD. Le présupposé institutionnel est que ces productions apporteront des connaissances pléthoriques et précises sur les thématiques environnementales. En les examinant, on constate que leur registre scriptovisuel est fortement marqué par l'angle scientifique (figure I), c'est-à-dire par l'exposition de concepts scientifiques et/ou de protocoles et de résultats de recherche. Exprimant des relations à l'environnement comme objet de connaissances (Le Marec, 20 I I), elles regorgent en effet « d'images-savoirs »-pour reprendre la catégorie sémiotique d'Andrea Catellani (2016 : 84) : graphiques administrant la preuve du vrai en représentant « des relations entre des "choses" identifiées indiscutablement et admises comme telles » (Bertin cité in : Jacobi, 1987 : 120) ; schémas aux propriétés synoptiques, généralisatrices et objectivantes (Vézin, 1982) comme moyen de monstration logique d'un état des savoirs ou encore imageries scientifiques spécifiques comme observations ou modélisations du réel, dont la caractéristique commune est de ne pouvoir être figurées que grâce aux technologies employées lors du travail de recherche. Ce dernier est d'ailleurs souvent illustré par des photographies de chercheurs, en laboratoire ou sur leur terrain.

Cette énonciation de l'expertise scientifique montre une maîtrise de la gestion des questions environnementales et énonce un savoir sur l'environnement. Or, si elle permet de rendre compte d'avancées de recherche et d'expliquer des phénomènes, il n'est pas rare que les clés de lecture, comme les légendes, permettant au « profane » d'accéder totalement à l'interprétation scientifique des éléments monosémiques présentés ne soient pas toujours explicitées voire même données. Dans l'hybridation entre médias de vulgarisation et outils de valorisation de la recherche inhérents à la communication scientifique publique (Fayard, 1988), la connotation scientifique peut supplanter la dénotation scientifique, de sorte que si, d'un point de vue communicationnel, l'énonciation d'une scientificité est en correspondance avec les attentes de l'institution scolaire, il en découle un malentendu du point de vue éducatif. Manifestement, ces médias ne répondent pas à la prétention didactique que la légitimation scientifique leur accordait pourtant. L'autorité éditoriale de leur producteur aura suffi pour que soient anticipés des contenus exclusivement centrés sur la diffusion de connaissances. Ce hiatus s'explique probablement par la confiance un peu naïve que les cadres de l'EN accordent aux lieux de production des savoirs scientifiques et aux présupposés

\footnotetext{
${ }^{12}$ EN 17 signifie $17^{e}$ cadre de l'EN interrogé.
} 
positivistes quant à leurs communications. II y aurait là aussi comme la manifestation de la subordination de l'École aux instances académiques scientifiques dont elle dépendrait dans la mesure où sa fonction est d'incorporer progressivement les savoirs qui en sont issus. Mais cette forme d'allégeance face à l'expertise n'est qu'une des facettes des logiques de légitimation à l'œuvre.

\section{L'énonciation de l'injonction ou les attentes normatives implicites de l'institution scolaire}

La légitimation sur la base de l'expertise a aussi été invoquée pour expliquer le choix des cadres de retenir massivement des expositions de l'Ademe ${ }^{13}$ (Zwang, 2016) : « Parce qu'on estime qu'à l'Ademe c'est institutionnel, et donc pour nous, on n'a pas à interroger les contenus de quelque chose qui vient directement d'un ministère [..... On n'ira pas voir des experts pour leur demander ce qu'ils pensent des experts de l'Ademe » (EN 6). Or, la fonction principale de cette agence environnementale est de produire des campagnes de communication gouvernementales pour susciter des changements de comportements (Jalenques-Vigouroux, 2006) à travers un positionnement se revendiquant d'une neutralité sociale : la cible est un individu générique, sans caractéristique sociale, dont les comportements sont l'objet principal des communications (Comby, 20I3). Les campagnes légitimées pour l'École sont concentrées sur deux thématiques : l'énergie et les déchets, dont elle produit et coproduit $74 \%$ des expositions. Son énonciation des thèmes est focalisée sur des angles d'approches comportementaliste et comptable (figure I) - aspect pécuniaire déjà mis en évidence par Béatrice Jalenques-Vigouroux (2006) - à travers trois figures principales : le dessin, la liste et le tableau.

Première figure, statistiquement liée de façon robuste à l'Ademe (Zwang, 2013), le dessin constitue l'expression principale de ses communications, jusqu'à s'étendre aux expositions sur les déchets qu'elle a coproduites. Leur opérativité communicationnelle tient à une simplification et une essentialisation (Drouin, 1987). Ainsi le message comportementaliste se voit-il renforcé par sa fonction réductrice. Représentés de façon ludique pour attirer l'attention, des personnages agissants matérialisent des comportements caricaturaux, conformes ou contraires à des prescriptions, qu'ils soient des incarnations du « héros anonyme » (Gagnebien, Jalenques-Vigouroux, 20ll), individu vertueux et reconnu comme tel, ou, à l'opposé, des représentations de l'antihéros qui, implicitement et par contraste, placent le visiteur en potentiel héros se conformant aux normes attendues. La

\footnotetext{
${ }^{13}$ Sous la tutelle de trois ministères - le ministère de l'Écologie et du Développement durable, celui de I'Industrie et des Finances et celui de la Recherche (Jalenques-Vigouroux, 2006 : I I5) -, l'Ademe est un établissement public à caractère industriel et commercial reconnu statutairement par le Code de l'environnement comme intervenant dans le domaine de la protection de l'environnement dans les champs de la qualité de l'air, de la limitation et du traitement des déchets, de la réalisation d'économies d'énergie, du développement des technologies propres et de la lutte contre les nuisances sonores.
} 
deuxième figure est la liste. Souvent conjuguées à la première personne pour impliquer directement le lecteur, des phrases successives énumèrent les attitudes à adopter.Au-delà du contenu, la mise en liste procède d'une organisation graphique qui ordonne la pensée tout en ayant des propriétés mnémotechniques (Goody, 1977). Chaque prescription ainsi individualisée contribue à intégrer chaque tâche à réaliser comme une évidence. Enfin, le tableau, troisième figure, donne encore davantage de corps à l'incitation normative. Son « caractère bidimensionnel et figé » (ibid. : I I I) qui permet d'« assigner à chaque élément une position unique qui définit sans ambiguïté et en permanence sa relation aux autres » (ibid. : I33) catalyse une pensée classificatoire. Sous la forme de tableaux scindés en deux colonnes, cette représentation spatiale de l'écrit permet de marquer des oppositions très claires combinées de façon synoptique : c'est une incarnation visuelle de la dichotomie conceptuelle entre «bons » et « mauvais » gestes. La simplification qui en résulte a pour objectif d'orienter le visiteur vers le bon comportement à adopter, sous un habillage de rationalité présentant des choix.

Dans les textes officiels de l'EDD, l'EN se montre très ambiguë quant au comportementalisme : elle se défend d'inculquer des gestes, en affirmant que l'EDD doit s'ancrer dans une pensée complexe permise par des approches systémiques, tout en inscrivant dans la première circulaire qu'elle « est fondée sur l'acquisition de connaissances et de comportements » (MEN 2004). On peut donc émettre l'hypothèse selon laquelle l'institution scolaire attend dans les expositions de l'Ademe non un discours d'expert comme elle le prétend mais un discours normatif qui pourrait remplir le rôle qu'elle-même ne saurait assumer pour des raisons d'acceptabilité sociale tant on imagine mal aujourd'hui qu'elle revendique ouvertement une pédagogie exclusivement transmissive d'inculcation de pratiques et de valeurs comme au temps de l'instruction civique. À travers la légitimation de cette autorité éditoriale et des ressorts de son énonciation injonctive se noue alors un continuum entre des services de l'État pour gérer la population dès le plus jeune âge, dans une forme de « gouvernementalité » (Foucault, 1994) de la sphère privée.

\section{De l'énonciation des bonnes intentions ou le politiquement correct de la légitimation institutionnelle}

La légitimation institutionnelle est la (faible) part de légitimation qui repose sur les objectifs éducatifs de l'École, c'est-à-dire sur leur conformité avec les textes en vigueur, en particulier sur leurs aspects axiologiques (Zwang, 20 l6). De cette logique résulte l'exclusion systématique des productions des associations environnementales dont l'institution présuppose qu'elles pourraient y laisser transparaître un habitus naturaliste (Waldvogel, 20II) de défense de l'environnement pétri de valeurs biocentriques et contre lequel l'EN s'est très clairement positionnée (Zwang, Girault, 2012) : « [Les associatifs] ont tout centré sur la nature et l'environnement. [...] Mais nous avec les textes fondateurs, du BO [Bulletin officiel] on est passé à l'éducation au 
développement durable. C'est-à-dire [à] tous les volets, pas que l'environnement » (EN 12). Ainsi les producteurs qui intègrent le développement durable en tant que nouveau paradigme des problématiques socio-environnementales se voientils aisément légitimés par le réseau de documentation pédagogique avec comme résultante que cette thématique est la plus importante du corpus (27 expositions sur 110) : « J'aurais envie de dire qu'en fait c'est les thématiques qui font qu'on s'intéresse... enfin, qu'on s'oriente plus vers des organismes ou des associations de développement durable et de solidarité » (EN 4).

En effet, parmi les productions retenues, figurent celles des associations à mission de solidarité internationale (Peuples solidaires et Valmy) et d'éducation à la solidarité (Orcades et Intercultural network for development and peace - INDP) qui se sont très tôt emparées de cette thématique et dont les titres célèbrent l'avènement du modèle de développement : « Les enjeux du développement durable » (Orcades, 200I) $)^{14} ;$ « Le développement durable, un jeu d'enfants!» (Peuples solidaires, 2002) ; «Le développement durable, une autre vision du monde » (Valmy, 2005) ; « Développement durable et justice sociale en Inde » (INDP, 2008). Elles présentent comme caractéristique l'énonciation de leurs thèmes, essentiellement sociaux (citoyenneté, éducation, égalité hommes-femmes, équité sociale et pauvreté, santé, solidarité locales et internationales) selon deux angles d'approche (figure I) : un angle d'ordre politique, qui renvoie aux actions des sociétés et des individus dans l'exercice de leurs droits et devoirs et un angle appelé « grands principes » car il fait référence à des principes internationaux (de l'action 2 I, de précaution) ${ }^{15}$ et/ou à des déclarations d'intention.

L'énonciation du politique est donnée à voir par un ensemble d'« images-preuves » (Catellani, 2016 : 84) à valeur testimoniale. La plupart du temps, il s'agit de photographies montrant les conditions de vie des populations de pays du Sud, en particulier celles des femmes et enfants dont on rappelle les droits internationaux, mais aussi de toute donnée permettant d'ancrer ce témoignage dans une réalité sociale. L'intention est la monstration pour une prise de conscience, parti pris étendu aux thèmes environnementaux abordés. Par exemple, un panneau sous-titré « $\mathrm{Au}$ Cameroun, des forêts sont en danger » (Peuples solidaires, 2002) expose le drapeau du pays, des données chiffrées relatives à la démographie et au développement comme l'espérance de vie et le taux de scolarisation, qui informent sur sa situation économique et sociale, puis deux images côte à côte (figure 2). À gauche, une photographie montre un engin orangé déplaçant avec une mâchoire mécanique d'imposants troncs coupés de couleur fauve, qui, rognée de façon circulaire, est encadrée par une forêt luxuriante et verte : la superposition des deux images

\footnotetext{
${ }^{14}$ « Les enjeux du développement durable » est la première exposition sur le développement durable de tout le corpus.

15 Les conférences des Nations unies sur l'environnement et le développement énoncent des principes et des objectifs de mise en œuvre de la politique internationale en matière de développement durable. L'action 2 I (ou agenda 2I) est un document qui a longtemps été emblématique de ce point de vue. Constitué de 40 chapitres, il a été adopté par 173 chefs d'État lors du sommet de Rio de 1992.
} 
symbolisant la trouée de la déforestation dans la végétation. À droite, un transat de jardin en teck détouré est décontextualisé, inséré sur un fond laiteux pour signifier le résultat de la coupe dite à blanc. La moitié inférieure du document est occupée par un grand encadré mettant en scène, par des guillemets, la retranscription de la parole d'un « responsable d'une association camerounaise » sur le rôle d'entreprises françaises, non citées, dans la coupe illégale d'essences précieuses pour le mobilier d'extérieur.Si l'ensemble manque de références précises, probablement pour que la dénonciation ne verse pas dans l'accusation, l'énonciation rend compte de l'internalisation d'un rapport politique à l'environnement. Mais sa portée se voit d'emblée atténuée par la solution au problème soulevé, donnée dès le titre du panneau : « Le développement durable vise à protéger et respecter l'environnement ».

Figure 2. Panneau 6 de l'exposition « Le développement durable, un jeu d'enfants » (Peuples solidaires, 2012) ) $^{16}$

En effet, l'énonciation des bonnes intentions se juxtapose de façon systématique à la figuration du vulnérable (humain et non humain), le plus souvent en la concentrant dans la titraille des documents - « La protection de l'environnement pour tous... maintenant! » que surplombent, en exergue, les principes 4 et 6 de l'agenda 2I (Valmy, 2005) ou encore « Il est nécessaire d'être le changement que l'on voudrait dans le monde. M. K. Gandhi » (INDP, 2008). Les « grands principes » s'appuient sur une intertextualité personnifiée par des citations d'auteurs faisant autorité (KriegPlanque, 2010) ou proclamée en référence au corpus onusien (en particulier au plan d'action pour le $21^{\mathrm{e}}$ siècle ${ }^{17}$ et au rapport Our Common Future - Brundtland, 1987 -, d'où est tirée l'antienne ${ }^{18}$ servant à définir le développement durable et présente dans $50 \%$ des expositions sur la thématique), dans une rhétorique de la déclaration d'intentions qui permet de tenir des propos dogmatiques en faveur de l'avènement d'un monde meilleur. Ce caractère doctrinal se parachève par la présentation de thèmes sous

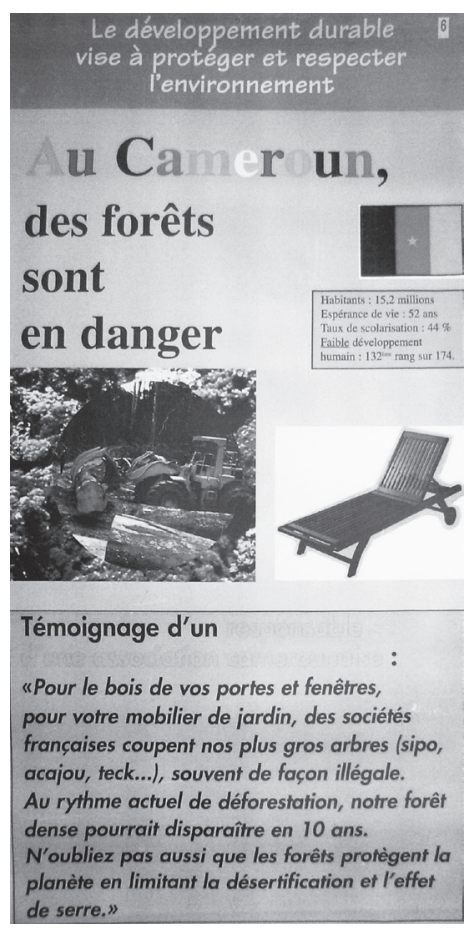

\footnotetext{
${ }^{16}$ Photographie obtenue gracieusement grâce à Jérôme Martin de l'association Kurioz (association d'éducation populaire de solidarité internationale, paix et développement durable).

17 Accès : http://www.un.org/french/ga/special/sids/agenda2 I/action0.htm. Consulté le 24/I I/20 I7.

18 « Le développement durable est un développement qui répond aux besoins du présent sans compromettre la capacité des générations futures de répondre aux leurs » (Brundtland, 1987 : en ligne).
} 
l'angle de l'exemplarité, typique des expositions sur le développement durable (Zwang, 20I3), par lequel ces principes moraux et/ou politiques se voient promus via des cas (casus) réels qui témoignent de leur mise en pratique : « Kérala, la force de l'ambition (Inde) » (Orcades, 200 I) est un exemplum d'une « casuistique morale contemporaine » (Seurrat, 2009 : 245), soit un modèle qui enjoint à la prise d'initiatives identiques.

En légitimant les expositions d'associations humanistes, l'EN s'inscrit dans un politiquement correct où les enjeux se voient énoncés sans risque ni sur les sujets sociaux abordés, essentiellement bornés aux pays du Sud, ni sur les aspects économiques car la remise en question des modèles actuels s'efface derrière la quête du développement durable, ni enfin sur les aspects environnementaux car leur sociocentrisme (Fortin-Debart, 2004), connu et attendu de l'institution, les éloigne des controverses sur notre rapport éthique à la nature. En définitive, leurs prises de position politiques sont neutralisées par un périmètre d'acceptabilité qui est à la mesure des idéaux énoncés et dont on peut supposer que l'EN voudrait qu'ils nourrissent les esprits des élèves. Cette hypothèse du penchant pour l'instruction en EDD est d'ailleurs corroborée par les ressorts de la légitimation politique.

\section{L'énonciation de l'instruction en jeu dans la légitimation politique}

J'ai nommé légitimation politique (Zwang, 2016) la légitimation éducative qui résulte directement de l'exercice d'un pouvoir, selon ce qu'un cadre de l'EN a qualifié de « loi d'airain » (EN 9) pour appuyer très clairement sur son penchant oligarchique. Le cabinet du ministre est approché par des producteurs, en l'occurrence GoodPlanet et Deyrolle, et il décide de s'y associer. Puis, une chaîne d'exécution s'organise, de la coproduction au niveau de la Direction générale de l'enseignement scolaire à la diffusion dans les réseaux de documentation. Le résultat est que le logotype de l'institution scolaire se voit directement apposé sur les expositions, sceau officiel qui reconnaît scripturalement et symboliquement la ressource légitime pour éduquer les élèves au développement durable.

Le « kit pédagogique » réalisé en 201 I avec Deyrolle est constitué de 10 planches, chacune sur un thème différent ${ }^{19}$. II a été distribué gratuitement dans 5000 écoles de trois académies (Rennes, Caen et Rouen) à la suite d'un accord au niveau national. La proposition de Deyrolle a trouvé un écho dans le projet politique de retour aux fondamentaux porté par le ministre de l'Éducation nationale Luc Chatel (2009-20 I2) : « Le prince de Broglie, qui a repris Deyrolle [...] avait cette idée de

\footnotetext{
${ }^{19}$ Les titres des 10 planches sont : « L'abeille, l'homme et le miel »; « La biodiversité - les services écologiques »; « Le changement climatique »; « Le développement durable »; « Les déchets »; « Les énergies renouvelables »; « La gestion durable de la forêt »; « Les gestes responsables »; « Le cycle du papier »; « Santé et climat ».
} 
repartir des fameuses planches Deyrolle, qui avaient fait le succès de sa maison, mais aussi de l'École de la $\| I^{\mathrm{e}}$, IV $\mathrm{V}^{\mathrm{e}}$ République, et évidemment, c'était quelque chose d'assez séduisant, dans la mesure où nous, nous essayions, en accord avec la modernité, de repartir sur des schémas très classiques, en matière d'enseignement, à l'École primaire, autour de l'apprentissage des fondamentaux » (EN I I).

Dans cette alliance, l'énonciation éditoriale de Deyrolle, la même qu'il y a un siècle, a joué un rôle déterminant : même planche rigide, même fond blanc encadré d'un liseré noir, alors typique de l'imagerie scolaire de la fin du XIX et du début du $x x^{e}$ siècle (Renonciat, 201 I), même typographie à empattement, même position du titre, centré en haut, du numéro, en haut à droite, et de la signature, centrée en bas. Au milieu, se déploient des blocs, paragraphes courts et compacts, titrés en majuscules et accolés aux dessins qu'ils commentent. Autant d'éléments inchangés capables de résonner avec des souvenirs de cadres de l'EN, voire d'éveiller la nostalgie d'une École plus disciplinée ou moins informatisée peut-être, dans un contrat de lecture qui vise directement ces cadres et non l'élève d'aujourd'hui.

Les dessins sont les points d'orgue de cette réminiscence de l'enseignement par l'image souhaité dès 1880 par Jules Ferry (Mœglin, 2004) et dont la palette s'étendait alors du croquis naturaliste à la mise en scène du labeur, de la famille, du terroir et des bonnes mœurs, dans une forme d'« esthétique hygiéniste » (Méneux, 2006) que ne saurait démentir le panneau édité par Deyrolle, cosigné en 20 I par l'EN et intitulé « Les gestes responsables ». Lorsque l'EN a apposé son propre logotype aux planches pour marquer son travail éditorial - qui s'est essentiellement centré sur le contenu des textes en ôtant toute dissonance sur l'éthique environnementale à faire prévaloir (Zwang, 2016) -, elle a fait sienne une énonciation de l'instruction aussi éloquente sur les desseins pédagogiques du cabinet ministériel que les nombreux textes publiés au Bulletin Offıciel, en ce sens que le rapport à l'éducation à l'environnement qui y transparaît n'est pas celui des approches et méthodes systémiques pourtant prônées dans ces derniers mais plutôt la réduction à une inculcation de savoirs et de (bonnes) pratiques.

\section{Des rapports triviaux instituant, dans l'École, une dépolitisation des problématiques socio-environnementales}

Même s'ils lient dans leur singularité des énonciations particulières à des représentations contextualisées, les différents contrats de communication précédemment mis en évidence conduisent tous à la légitimation de médias éducatifs qui éludent les controverses. De fait, les cadres de l'EN sélectionnent des productions d'instituts de recherche scientifique dont l'épistémologie internaliste des sciences élargit peu les questions socio-environnementales aux aspects sociaux ou politiques. Ils s'orientent vers des producteurs humanistes qui lissent les tensions 
politiques et les injustices sociales derrière l'angélisme des déclarations onusiennes. Enfin, ils privilégient des institutions à l'origine de campagnes de communication publiques faisant «du bruit sans faire de vague »(Comby, 2013 : en ligne) en omettant les responsabilités des structures collectives ou de groupes sociaux aisés au profit d'une individualisation par des gestes quotidiens. Ainsi est-ce une bienpensance environnementale et sociale ne donnant médiatiquement pas de prise à une éducation à l'environnement socialement critique qui se voit privilégiée.

En s'en remettant aux intentions de communication des institutions, elle confie les énoncés à des autorités éditoriales dont les politiques textuelles sont inoffensives (dans le sens de sans offenses, sans heurts) et/ou normatives du point de vue des enjeux socio-environnementaux. II en va de même lorsqu'elle incarne cette autorité dans des opérations éducatives d'ampleur nationale, en se donnant à voir soit comme prescriptrice d'une instruction environnementale avec Deyrolle ou, avec GoodPlanet, comme une institution sans frontières, ouverte sur la nature et les problématiques socio-environnementales contemporaines, mais dont les images si esthétisantes transcrivent des relations au monde bornées par le beau. Dans les deux cas, les énonciations éditoriales laissent transparaître des partis pris communicationnels qui, dans leur dimension symbolique, s'avèrent contraires aux prescriptions relatives aux débats et à l'éducation au choix. Or, dans les jeux de légitimation se déroulant aux portes de l'École, les attributs scripturaux de l'objet communicationnel, et en particulier, l'apposition d'un logotype de l'EN contribuent à le charger de valeur éducative. Ainsi le rapport de l'École à la trivialité, et plus particulièrement à la dimension communicationnelle des controverses (Le Marec et Babou, 2015), consiste-t-il à neutraliser le politique. Les finalités éducatives repérables en matière d'éco-citoyenneté sont donc finalement moins l'expression d'une vitalité démocratique de la Cité que celle d'une nouvelle forme de civisme.

\section{Conclusion}

L'analyse communicationnelle de la mise en circulation des expositions itinérantes pour l'EDD permet de mettre en évidence que les dissonances entre contenus prescrits dans les textes et contenus légitimés dans ces médias tirent leur origine dans les contrats implicites qui lient les attentes de l'EN aux intentionnalités communicationnelles des autorités éditoriales. Ce rapport trivial que l'École établit avec les productions médiatiques relatives aux problématiques socioenvironnementales est à considérer dans la mesure où il structure une circulation de savoirs, valeurs et représentations non pluraliste et non contradictoire dans l'espace scolaire. En effet, dans une perspective éducative visant à remettre en cause les relations actuelles entre les humains et leur environnement, la portée de ces communications est à interroger dans la mesure où elles deviennent des sources de savoir une fois territorialisées dans cet espace, investi comme lieu de culture porteur de savoirs et de valeurs. 
De façon plus générale, ces travaux esquissent la nécessité de décrypter encore plus avant comment, par les formes de communication, et plus particulièrement par la manière dont le registre scriptovisuel opère, s'instituent, en milieu éducatif, certains rapports à la nature au détriment d'autres. Autrement dit, il semble que considérer les idées circulant à l'École dans leur dimension communicationnelle du point de vue symbolique et de la politique des textes, c'est-à-dire bien au-delà des approches par les contenus de savoirs (controversés ou non) plus traditionnelles en sciences de l'éducation constitue un enjeu en éducation relative à l'environnement.

\section{Références}

Boutaud J.-J., Verón E., 2007, Sémiotique ouverte. Itinéraires sémiotiques en communication, Cachan, Lavoisier.

Brundtland G. H., dir., 1987, Rapport de la Commission mondiale de l'environnement et le développement de l'ONU. Accès : http://fr.wikisource.org/wiki/Notre_avenir_à_tous__Rapport_Brundtland. Consulté le 24// I/2017.

Catellani A., 2016, « Sémiotique de la communication environnementale », pp. 77-93, in : Libaert T., dir., La Communication environnementale, Paris, CNRS Éd.

Comby J.-B., 2013, « Faire du bruit sans faire de vagues. Une analyse sociologique de la communication de l'État sur les questions climatiques », Communication, 31. Accès : http://communication.revues.org/4439.

D'Almeida N., Jalenques-Vigouroux B., 2007, « Dire le développement durable », Annales des Mines, série Responsabilité et environnement, 48, pp. 44-5I.

Davallon J., 1999, L'Exposition à l'œuvre. Stratégies de communication et médiation symbolique, Paris, Éd. L'Harmattan.

Deyrolle, I898, Catalogue de mobilier et de matériel scolaire pour les enseignements maternel, primaire, secondaire et supérieur, Paris, Deyrolle.

Drouin A.-M., 1987, « Des images et des sciences », Aster, 4, pp. I-31.

Fayard P., 1988, La Communication scientifique publique. De la vulgarisation à la médiatisation, Lyon, Chronique sociale.

Fortin-Debart C., 2004, Le Partenariat école-musée pour une éducation à l'environnement, Paris, Éd. L'Harmattan.

Foucault M., 1994, Dits et écrits, 1954-1988. III, 1976-1979, Paris, Gallimard.

Gagnebien A., Jalenques-Vigouroux B., 201 I, « Mise en récit du développement durable : comment le musée élabore ses expositions », pp. 23 I-248, in : Chaumier S., Porcedda A., dirs, Musées et développement durable, Condé-sur-Noireau, Documentation française.

Goody J., 1977, La Raison graphique. La domestication de la pensée sauvage, trad. de l'anglais par J. Bazin et A. Bensa, Paris, Éd. de Minuit, 1978.

Jacobi D., 2005, « Images originales et images de reproduction dans l'exposition », pp. 90- I27, in : Jacobi D., Lochot S., dirs, Images d'expositions, expositions d'images, Dijon, Office de coopération et d'information muséales. 
Jalenques-Vigouroux B., 2006, Dire l'environnement . Le métarécit environnemental en questions, thèse en sciences de l'information et de la communication, Université Paris-Sorbonne.

Jeanneret Y., 1999, « L'École et la culture triviale », pp. 7-23, in : Bentolila A., dir., École et modernités, Paris, Nathan.

Jeanneret, Y., 2008, Penser la trivialité, Cachan, Lavoisier.

Jeanneret Y., 2014, Critique de la trivialité. Les médiations de la communication, enjeu de pouvoir, Paris, Ed. Non Standard.

JeanneretY., Patrin-LeclereV., 2004, « La métaphore du contrat », Hermès, 38, pp. I 33- 440.

Krieg-Planque A., 2010, "La formule "développement durable" : un opérateur de neutralisation de la conflictualité », Langage et société, I34, pp. 5-29.

Le Marec J., 2007, Publics et musées. La confiance éprouvée, Paris, Éd. L'Harmattan.

Le Marec J., 20 I I, « L'environnement et la participation au musée : différentes expressions culturelles des sciences », Hermès. La Revue, 61, pp. I67-174.

Le Marec J., Babou I., 20 I 5, « La dimension communicationnelle des controverses », Hermès, 73, pp. $111-121$.

Le Marec J., Topalian R., 2003, « Énonciation plurielle et publication de la parole du public en contexte muséal : le cas de "la tribune des visiteurs" », Communication et langages, I 35, I, pp. $12-24$.

Libaert T., dir., 2016, La Communication environnementale, Paris, CNRS Éd.

Méneux C., 2006, « L'art social au tournant du siècle », Arts et sociétés, Lettre du séminaire, 12. Accès : http://www.artsetsocietes.org/f/f-meneux.html. Consulté le 24/I I/20 17.

Ministère de l'Éducation nationale (MEN), 2004, « Généralisation d'une éducation à l'environnement pour un développement durable », circulaire $n^{\circ} 2004-$ I 10.

Mœglin P., 2004, Outils et médias éducatifs. Une approche communicationnelle, Grenoble, Presses universitaires de Grenoble.

Renonciat A., 20 I I, Voir-savoir. La pédagogie par l'image aux temps de l'imprimé , du XVle au $X X^{e}$ siècle, Futuroscope/Scérén-CNDP-CRDP.

Seurrat A., 2009, Les Médias en kit pour promouvoir la diversité. Étude de programmes européens de formation aux médias destinés à "l lutter contre les discriminations 》 et " promouvoir la diversité », thèse en sciences de l'information et de la communication, Celsa Paris-Sorbonne.

Souchier E., 1998, « L'image du texte. Pour une théorie de l'énonciation éditoriale », Les Cahiers de médiologie, 6, pp. I37-| 45.

Souchier E., 2007, « Formes et pouvoirs de l'énonciation éditoriale », Communication et langages, 154, I, pp. 23-38.

Véron E., 1984, «Quand lire c'est faire : l'énonciation dans le discours de la presse écrite », Sémiotique II, Paris, Institut de recherches et d'études publicitaires.

Vézin J.-F., 1982, «Apport informationnel des schémas et des énoncés et activités de mise en correspondance », Enfance, 35, 5, pp. 35I-362.

Waldvogel C., 201 I, Imposer « l'environnement 》: le travail révélateur des associations alsaciennes (1965-2005), Strasbourg, Presses universitaires de Strasbourg. 
Zwang A., 2013, Les Expositions comme médias éducatifs pour l'Éducation au développement durable : une légitimation en tension entre cadres prescriptifs et images des concepteurs, thèse en muséologie, Muséum national d'histoire naturelle.

ZwangA., 20 I 6, «La légitimation d'expositions itinérantes pour l'éducation au développement durable : des objectifs de l'École à ceux des producteurs », Recherches en didactique des sciences et des technologies, 13, pp. 21 -49.

Zwang A., Girault Y., 2012, « Quelle(s) spécificité(s) pour l'Éducation au Développement Durable? », Spirale, 50, pp. 181-195. 